\title{
SURFACE MICROMACHINED LEAKAGE PROOF PARYLENE CHECK VALVE
}

\author{
Jun Xie, Xing Yang, Xuan-Qi Wang, and Yu-Chong Tai \\ Electrical Engineering, 136-93 \\ Caltech Micromachining Laboratory \\ California Institute of Technology \\ Pasadena, CA, 91125 , U.S.A.
}

\begin{abstract}
We report here a surface micromachined leakage proof Parylene MEMS check valve that has nearly ideal performance. This new check valve has a unique design that its valve membrane has a sealing ring directly deposited on, and hence in direct contact with, the gold-coated valve seat. The adhesion between Parylene and gold is studied along with the reduction of adhesion of the sealing ring to the valve seat when self-assembled monolayer (SAM) coatings are used on gold. Testing results clearly demonstrate the effectiveness of SAM coating. Experiments show that the valves have no observable leakage in the reverse flow direction up to $30 \mathrm{psi}$ and a cracking pressure less than $1 \mathrm{psi}$ in the forward flow direction. Integration of this valve with microchannels in a microfluidic system is also demonstrated. Testing shows the in-channel check valve also has nearly ideal performance under both forward and reverse flow.
\end{abstract}

\section{INTRODUCTION}

In the past few years, many MEMS fluidic handling devices such as valves and pumps. have been developed with the ultimate goal of achieving integrated microfluidic systems for chemical and biological applications. As a fundamental component in such a system, a check valve is a passive device that allows fluid flow in forward direction and prevents the flow in the reverse direction. An ideal check valve should have zero cracking pressure and zero flow resistance in the forward direction and infinite flow resistance in the reverse direction.

There are many published MEMS check valves [1-3]. Various materials (including silicon, silicone, polyimide, and metal) along with various technologies have been used to construct check valves. Previously we developed surface micromachined check valves using Parylene, a CVD thin-film polymer, as the structural material with photoresist as the sacrificial layer. We reported that Parylene has the advantages of low modulus, good sealing, and good biocompatibility.

Although our previous Parylene check valve [2] shows good performance, it has a gap created by the sacrificial layer between valve membrane and valve seat. Fluid can then leak through this gap in the reverse flow direction, which could have even more serious leakage problems when the check valve is integrated within microchannels. In a microchannel, the pressure drop across the check valve may be too small to close the valve. It is the goal of this work to achieve a check valve with zero leakage in the reverse flow direction even under very low reverse pressure drop and demonstrate it inside a microchannel. As a result, we propose a new check valve design that has a sealing structure that completely eliminates the gap between the sealing plate and the valve seat. By using this method, the in-channel check valve only needs 2 layers of Parylene. The new sealing structure is a Parylene sealing ring directly deposited onto valve seat surface. Because of the direct deposition of Parylene on the valve seat surface, the sealing plate needs to be pneumatically released from the valve seat before normal operation. It is found that this initial release pressure has a great effect on the performance of the check valve. To reduce the release pressure, we have put gold on the valve seat surface and used self-assembled monolayer (SAM) coating for gold [4] to further reduce the adhesion between Parylene and gold. As shown in the following, the testing results prove the effectiveness of SAM coating on gold. Flow characteristics also show that both out-of-channel check valve and in-channel check valve have nearly ideal performance in both forward and reverse directions.

\section{DESIGN}

Shown in Figure 1, the design for the check valve outside a channel is a Parylene disk suspended by four tethers. Unlike our previous check valve, there is a sealing ring structure on the edge of the Parylene disk. This sealing ring structure is formed by patterning a ring window in the photoresist sacrificial layer resulting in direct deposition of Parylene in the ring area. With such a sealing structure, there is no initial gap between the sealing plate and the valve seat. This no-gap design helps to achieve leakage proof in the reverse flow direction even under lowpressure drop. The radius of the valve sealing membrane varies from $60 \mu \mathrm{m}$ to $120 \mu \mathrm{m}$ and $5 \mu \mathrm{m}$ to $20 \mu \mathrm{m}$ for the width of the sealing ring.

After the check valve is fabricated, the sealing ring part of the valve membrane has to be pneumatically released from the valve seat before it can be used. It is found that this initial release pressure has a great effect on the performance of the check valve

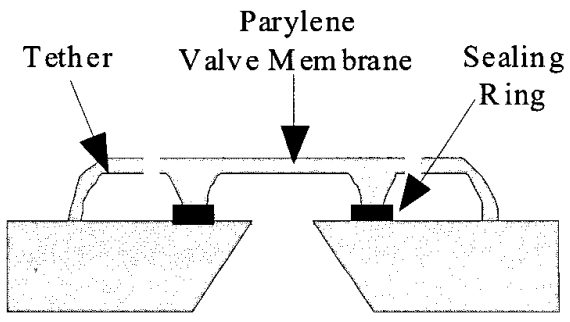

Figure 1. Conceptual Design of the Check Valve 


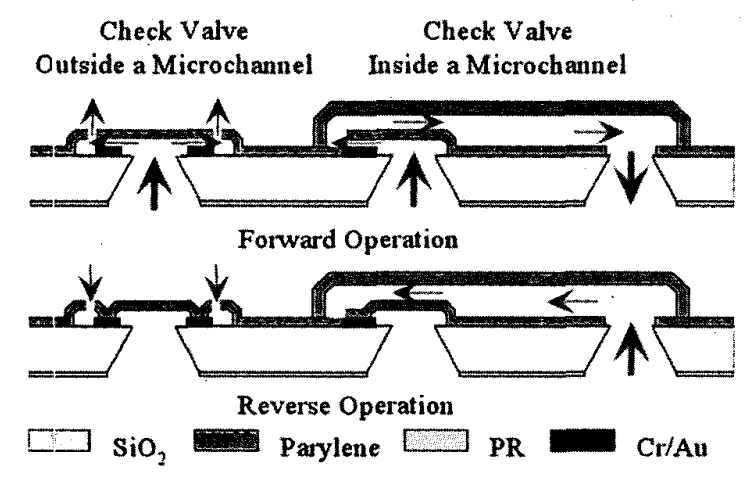

Figure 2. Check Valve Outside and Inside a Microchannel

because high initial release pressure may cause permanent plastic deformation in the valve membrane and then compromise the valve sealing. To solve this problem, one can use a surface with poor adhesion to Parylene under the sealing ring structure. Gold typically. has poor adhesion to Parylene compared to other surfaces, such as $\mathrm{SiO}_{2}$ and $\mathrm{Si}$. Experimentally, we found that the adhesion between Parylene and gold is still fairly strong so the large release pressure did damage the Parylene sealing membrane. As a result, we have used octadecanethiol SAM coating on gold. This SAM coating on gold is proved to be very effective in reducing the adhesion. Test results are shown in next section.

To integrate the check valve in a microchannel, some modification has to be made. In the case of check valve outside the microchannel, all the pressure is applied across the valve. But when the valve is placed inside a channel, the pressure drop across the valve is much smaller if the dimensions of the channel and the valve are similar. To increase the pressure drop, check valve with smaller dimensions should be used. In another design, a sealing arc (15 $\mu \mathrm{m}$ long and $60 \mu \mathrm{m}$ wide) instead of the sealing ring is used. The cesign of the check valve inside and outside a microchannel is shown in Figure 2.

\section{FABRICATION}

The check valves are fabricated using surface micromachining technology with Parylene-C as the structural layer and $A . Z$ photoresist as the sacrificial layer.

Figure 3 shows the fabrication process for the check valve. The process starts with 4" silicon wafers. First, a layer of $1.6 \mu \mathrm{m}$ thick thermal oxide is grown on both sides of the wafers. Etching windows are patterned on the wafer backside. $\mathrm{KOH}$ is used to etch holes in the silicon substrate until a $15 \mu \mathrm{m}$ thick silicon membrane is left on the front side of the wafer. These holes serve as the fluid inlet/outlet for the valve. Then a layer of $100 \AA \mathrm{Cr}$ and $2000 \AA \mathrm{Au}$ is thermally evaporated on the wafer front side. The tri-layer of $\mathrm{Au} / \mathrm{Cr} / \mathrm{SiO}_{2}$ is patterned to form the sealing ring area where the Parylene sealing ring is directly deposited. The wafers are then etched for one minute in $\mathrm{BrF}_{3}$ vapor to roughen the silicon surface. During the $\mathrm{BrF}_{3}$ etching, the silicon surface is etched away by $0.5 \mu \mathrm{m}$.

This $\mathrm{BrF}_{3}$ roughening step serves two purposes. First it helps to improve the adhesion of Parylene layer to the silicon substrate.

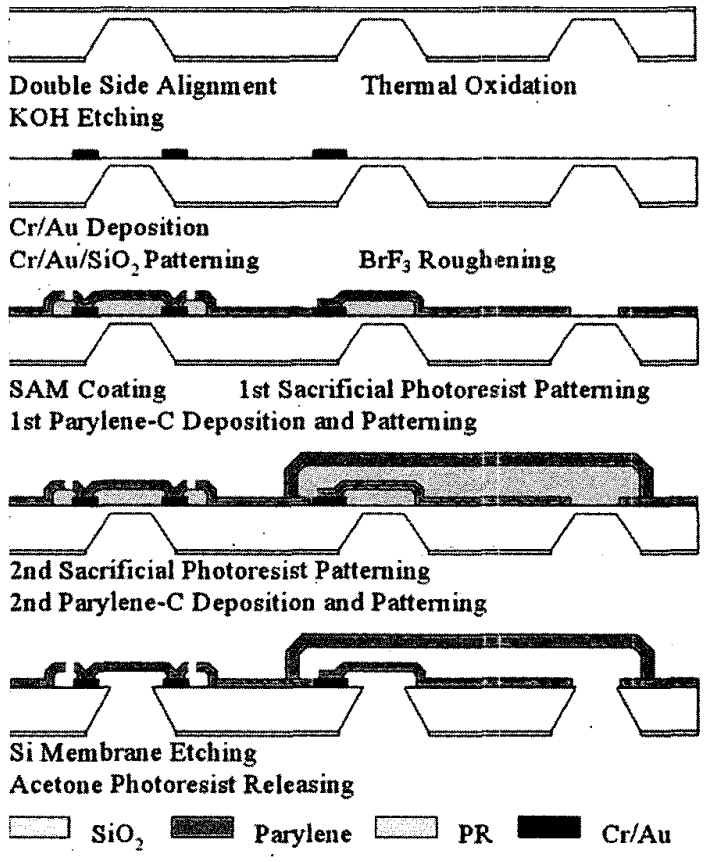

Figure 3. Check Valve Fabrication Process Flow

Adhesion promoter (OSI A-174) could not be used because of the presence of the photoresist sacrificial layer. Second, the area under the sacrificial photoresist layer is also roughened to reduce the stiction that may happen during the final acetone releasing of the photoresist sacrificial layer. For valves with large dimensions, an array of posts is fabricated in the valve membrane to further reduce stiction.

Before the SAM coating for gold, gold surface is cleaned in 200 mTorr oxygen plasma with $200 \mathrm{~W}$ power for 2 minutes. General instructions and principles on organic thiol SAM coating for gold are given in [4]. In our experiment, octadecanethiol is used as the coating material. $0.4 \mathrm{~g}$ octadecanethiol is dissolved in $800 \mathrm{~mL}$ ethanol $(\sim 2 \mathrm{mM})$. Then the wafers are immersed in the solution for 10 hours. After the wafers are taken out of the solution, they are air dried for 15 minutes and followed by a 30 seconds rinse in ethanol and a 10 minutes bake at $50^{\circ} \mathrm{C}$. Water contact angle measurement is used to monitor the coating process.

After SAM process, a $1 \mu \mathrm{m} \mathrm{AZ} 1518$ photoresist layer is spun and patterned to form the first sacrificial layer. This sacrificial layer separates the valve membrane from the valve seat except in the sealing ring region. A $0.5 \mu \mathrm{m}$ thick Parylene-C layer is deposited and then patterned by using oxygen plasma with $4 \mu \mathrm{m}$ thick soft baked AZ 4400 photoresist as the etching mask. Finally the valves are freed by etching away the remaining silicon membrane and overnight acetone releasing of the photoresist sacrificial layer.

To fabricate the check valve inside a microchannel, a second sacrificial layer of $8 \mu \mathrm{m} \mathrm{AZ} 4620$ photoresist is spun and patterned to form the channel pattern. Before the second Parylene-C deposition, the wafers are cleaned and roughened by oxygen plasma in order to increase the adhesion between the two 


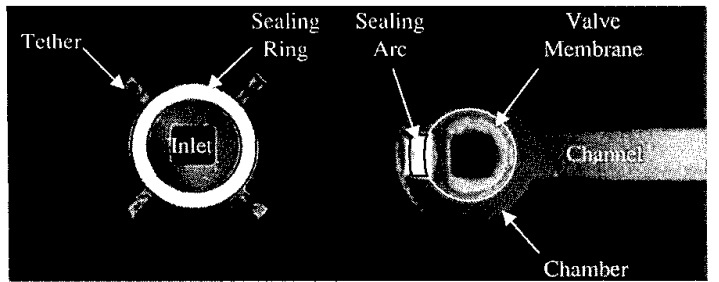

(a) Outside a Microchannel

(b) Inside a Microchannel

Figure. 4 Pictures of the Fabricated Check Valves

Parylene-C layers. A second layer of $5 \mu \mathrm{m}$ Parylene-C is deposited to form the channel wall. The final releasing steps are similar to the case of check valves outside the channel.

Shown in Figure 4 are pictures of fabricated check valves both outside a channel and inside a channel.

\section{EXPERIMENTAL RESULTS}

\section{SAM Coating Process}

Contact angle measurement of water on the gold surface is used to monitor the SAM coating process. Right after the oxygen plasma cleaning, gold and roughened silicon surface have contact angles of water less than $10^{\circ}$. After SAM coating, the contact angle on gold surface increases to $95^{\circ}$ while the contact angle on roughened silicon surface is still less than $10^{\circ}$. This confirms that SAM layer of octadecanethiol only coats gold surface as the principle of SAM coating implies. Also a measurement is made on gold surface before and after one cycle of photolithography process (spin coating, soft bake, exposure, development, rinse, and hard bake) and the contact angle on gold remains above $90^{\circ}$. Even after the whole fabrication process is finished the contact angle on gold still remains above $90^{\circ}$. This shows that the SAM coating of organic thiol on gold is very stable during the whole fabrication process.

Table 1 compares the release pressure of the valves with and without SAM coating. The initial release pressure is defined as pneumatic pressure needed to separate the valve membrane from the sealing ring contact and get a forward flow through the valve. During testing, it is found that if this release pressure is too high, it can cause plastic deformation on the Parylene valve membrane and thus seriously affect the valve performance. Table 1 shows that for the same design, the release pressure of those valves with SAM coating is on average 10 times smaller than those without SAM coating. This proves the effectiveness of SAM coating in reducing the adhesion between Parylene and gold.

Figure 5 shows a check valve that was initially released at a pressure of 27 psi. After releasing, the plastic deformation in the

\begin{tabular}{|c|c|c|}
\hline Release Pressure & R120W10 & R120W20 \\
\hline With SAM & $2 \mathrm{psi}$ & $2 \mathrm{psi}$ \\
\hline Without SAM & $18 \mathrm{psi}$ & $20 \mathrm{psi}$ \\
\hline
\end{tabular}

Table 1. Comparison of Release Pressure of Check Valves With and Without SAM Coating. The valves have radius of $120 \mu \mathrm{m}$ and sealing ring width of 10 and $20 \mu \mathrm{m}$.

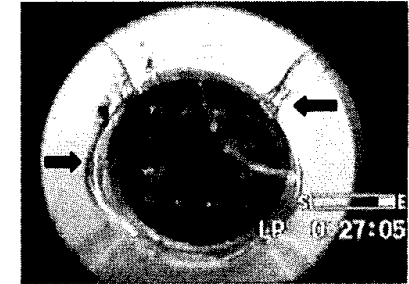

Figure 5. Plastic Deformation of the Valve Membrane and Tethers

Parylene membrane can be clearly seen as indicated by the arrows, and testing shows that the valve has severe leakage in the reverse flow direction.

\section{Valve Performance}

The flow performance of both types of check valve has been tested with air and water as the working fluid. A simple testing setup is built with compressed air as the pressure source. The applied pressure is controlled with pressure regulator $(0.1 \mathrm{psi}$ accuracy) and pressure gage ( $0.01 \mathrm{psi}$ accuracy). In the case of water testing, a column of water driven by the compressed air provides the working fluid. Flow rate is measured in a few different methods depending on the flow rate range. Air flow rates larger than $0.1 \mathrm{~mL} / \mathrm{min}$ and water flow rates larger than $1 \mu \mathrm{L} / \mathrm{min}$ are measured with commercial available flow meters. For smaller air flow rates, a calibrated pipette with a small water droplet is used. The flow rate can be obtained by measuring the droplet movement in a given time. Small water flow is measured in the similar way by following the movement of the water column front.

Figure 6 shows the measured air and water flow characteristics of a check valve outside a microchannel. Since the Parylene valve membrane is very thin $(0.5 \mu \mathrm{m})$, it tends to be plastically deformed or even break at the joint of the membrane and the tethers if the applied forward pressure is too large. Due to this, all the forward flow testing is performed at the pressure less than 10 psi. For reverse flow direction, valves are tested in both high-pressure region (up to $30 \mathrm{psi}$ ) and low-pressure region (down to $0.1 \mathrm{psi}$ ). Testing results in Figure 6 shows the valve has a forward flow rate of $2.5 \mathrm{~mL} / \mathrm{min}$ for air and $20 \mu \mathrm{L} / \mathrm{min}$ for water at 5 psi pressure. In the reverse flow direction, it shows no observable leakage up to $30 \mathrm{psi}$. The leakage is tested by observing the volume change of a water droplet on the valve outlet under the microscope. No significant volume change can be observed over 10 minutes.

The testing results of an in-channel check valve for both air and water are shown in Figure 7. Due to the presence of the microchannel $(80 \mu \mathrm{m}$ wide, $8 \mu \mathrm{m}$ high, and $7 \mathrm{~mm}$ long), the check valve has a much smaller flow rate compared to the check valve outside a channel. At 5 psi forward pressure, this valve has a flow rate of $3 \mu \mathrm{L} / \mathrm{min}$ for air and $150 \mathrm{~nL} / \mathrm{min}$ for water. In the reverse flow direction, leakage is tested in a similar way as described above and no observable leakage can be found up to $30 \mathrm{psi}$. 


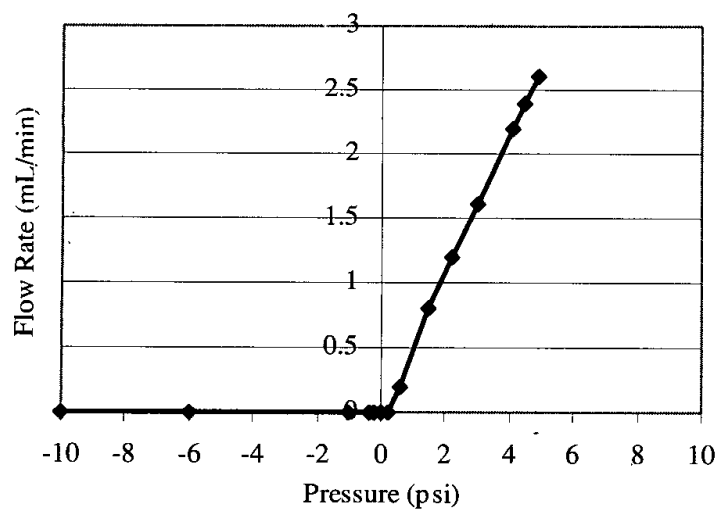

(a) Air

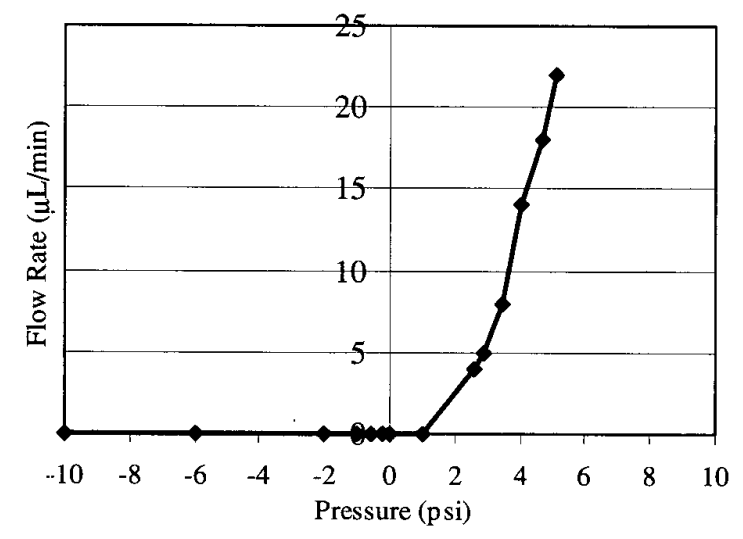

(b) Water

Figure 6. Air and Water Flow Characteristics of a Check Valve Outside a Microchannel

\section{CONCLUSIONS}

By using a unique sealing structure and surface micromachining technology, leakage proof Parylene check valves both outside and inside a microchannel have been successfully developed. The experimental results show the effectiveness of the design and the nearly ideal performance of the check valve under both forward and reverse flow. SAM coating is also demonstrated to be an effective way to reduce the adhesion between Parylene and gold. For future work, the integration of this check valve with other microfluidic devices, such as micro-nozzles, will be pursued.

\section{ACKNOWLEDGMENTS}

The authors would like to thank Dr. Terry D. Lee from Beckman Research Institute of the City of Hope for helpful discussion and support. Also the authors would like to thank $\mathrm{Mr}$. Yong $\mathrm{Xu}$ and $\mathrm{Mr}$. Trevor Roper for their assistance in the fabrication. This project is co-supported by NIH under Grant R01 RR06217-06 and NSF ERC Center of Neuromorphic Systems Engineering (CNSE).

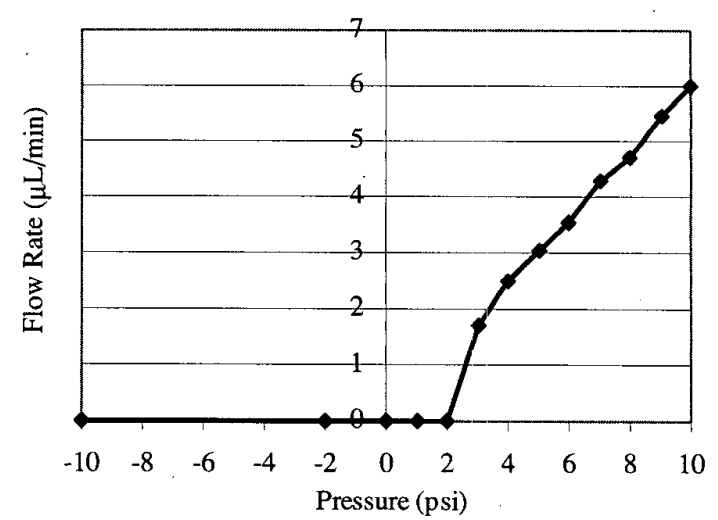

(a) Air

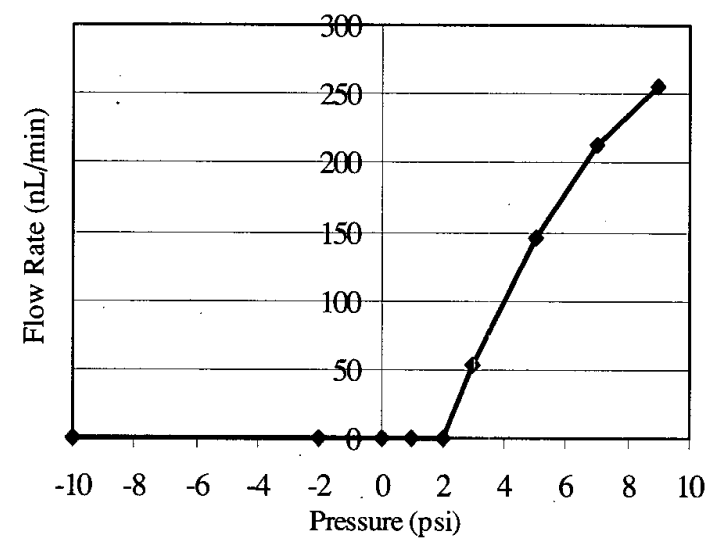

(b) Water

Figure 7. Air and Water Flow Characteristics of a Check Valve Inside a Microchannel

\section{REFERENCES}

1. S. Shoji, "Fluids For Sensor Systems," Topics in Current Chemistry, Vol.194, 163 (1998).

2. X. Q. Wang, Q. Lin, and Y. C. Tai, "A Parylene Micro Check Valve," Proc. of the $12^{\text {th }}$ IEEE MEMS 1999 Technical Digest, Orlando, Florida, 1/17-21/99, IEEE (1999), pp. 177-182.

3. X. Q. Wang and Y. C. Tai, "A Normally Closed In-Channel Micro Check Valve," Proc. of the $13^{\text {th }}$ IEEE MEMS 2000 Technical Digest, Miyazici, Japan, 1/23-27/00, IEEE (2000), pp. 68-71.

4. C. D. Bain, E. B. Troughton, Y. T. Tao, J. Evall J, G. M. Whitesides, and R. G. Nuzzo, "Formation of Monolayer Films by the Spontaneous Assembly of Organic Thiols from Solution onto Gold," J. Am. Chem. Soc., Vol.111, 321 (1989). 